\title{
Energy efficient transmission for LTE cellular system
}

\begin{abstract}
With the introduction of Long Term Evolution (LTE) cellular networks, the spectral efficiency should be maximized according to the required high data rate. However, any increase in spectral efficiency will lead to an undesirable increase in power consumption, and thus lower energy efficiency will be obtained. Therefore, a new problem in radio resource allocation is addressed to manage the bandwidth and power resources according to LTE and Green communication requirements. So far, the main objective of the most radio resource allocation algorithms is to increase the data rate without much consideration of the energy or power metrics. Some of them had to minimize the power consumption at a given data rate requirements. In this paper, we propose a multi-objective radio resource function which aims to maximize energy efficiency at a given data rate requirement. This function will consider dynamic subcarrier assignment to assign different subcarriers to different users. A multiuser orthogonal frequency division multiplexing (OFDMA) represents the physical layer of LTE system along with a single transmitting antenna to transmit the data in a frequency flat fading channel. It has been shown that the transmission bandwidth and modulation order have impacts on the energy efficiency.
\end{abstract}

Keyword: Energy efficiency; LTE; OFDMA; Resource allocation 